\title{
Rabaska
}

Revue d'ethnologie de l'Amérique française

[CENTRE FRANCO-ONTARIEN DE FOLKLORE]. Habiter le pays.

Inventaire du patrimoine de l'Ontario français. SERGE

SAINT-PIERREet al., sous la direction de GAÉTAN GERVAIS et

de JEAN-PIERRE PICHETTE. Sudbury, Prise de parole/Centre

franco-ontarien de folklore, 2001, 270 p. Illustrations, cartes,

index. ISBN 2-89423-127-X

\section{Bernard Genest}

\section{Numéro 1, 2003}

URI : https://id.erudit.org/iderudit/201615ar

DOI : https://doi.org/10.7202/201615ar

Aller au sommaire du numéro

\section{Éditeur(s)}

Société québécoise d'ethnologie

ISSN

1703-7433 (imprimé)

1916-7350 (numérique)

Découvrir la revue

Citer ce compte rendu

Genest, B. (2003). Compte rendu de [[CENTRE FRANCO-ONTARIEN DE FOLKLORE]. Habiter le pays. Inventaire du patrimoine de l'Ontario français. SERGE SAINT-PIERREet al., sous la direction de GAÉTAN GERVAIS et de JEAN-PIERRE PICHETTE. Sudbury, Prise de parole/Centre franco-ontarien de folklore, 2001, 270 p. Illustrations, cartes, index. ISBN 2-89423-127-X]. Rabaska, (1), 160-165. https://doi.org/10.7202/201615ar d'utilisation que vous pouvez consulter en ligne. 
[Centre fRANCo-OnTARIEN DE FolkLoRe]. Habiter le pays. Inventaire du patrimoine de l'Ontario français. Serge SaInT-PIERRE et al., sous la direction de GaÉtan Gervais et de Jean-Pierre Pichette. Sudbury, Prise de parole/Centre franco-ontarien de folklore, 2001, $270 \mathrm{p}$. Illustrations, cartes, index. ISBN 2-89423-127-X.

Ce livre est la synthèse d'un inventaire du patrimoine de l'Ontario français réalisé entre 1991 et 1994 par le Centre franco-ontarien de folklore de Sudbury. L'inventaire en question avait pour but de brosser un premier portrait du 
" paysage traditionnel » de l'héritage français en Ontario. Dans sa préface, le père Germain Lemieux, s. j., présente l'ouvrage en déclarant que « Même si le présent volume ne contient pas tous les résultats d'une recherche exhaustive et définitive, les lecteurs conviendront cependant que c'est un autre pas vers la connaissance d'un riche passé en vue de la construction d'un avenir digne de nos courageux ancêtres ». Le « nos » désigne, bien sûr, les Ontariens de souche francophone. Quant à l'historien Gaétan Gervais et à l'ethnologue Jean-Pierre Pichette qui ont conçu et dirigé le projet, ils témoignent dans l'avant-propos de ce que l'inventaire (et sans doute l'ouvrage qui en découle) servira « d'outil pédagogique pour la connaissance pratique du patrimoine bâti d'un village, d'une ville ou d'une région ou de l'ensemble de l'Ontario français; de jalon pour suivre l'évolution du patrimoine et entreprendre d'autres recherches; de guide pour la préparation d'itinéraires culturels, de levier pour le classement de nos biens culturels ; enfin de modèle pour les autres groupes ou cultures de l'Ontario qui voudraient étudier et promouvoir de la sorte leur propre patrimoine ". Le projet visait à identifier les lieux qui conservent des traces du passage des Franco-Ontariens, inventorier les sources documentaires qui les concernent, repérer le réseau institutionnel qui les encadre et monter des dossiers documentant leur vie matérielle, sociale et spirituelle. La méthodologie de base devait reposer sur des relevés photographiques de sites, d'objets et de personnes « témoins des traditions ». L'ouvrage se voulant une présentation synthèse de l'information recueillie sur le terrain, sa consultation devrait nous amener à juger de l'atteinte des résultats.

L'étude contient dix chapitres, dont neuf s'articulent autour des grandes divisions thématiques suivantes : patrimoine agricole, patrimoine religieux, architecture civile et domestique, patrimoine commercial et de services, patrimoine du domaine public et communautaire, patrimoine industriel, art populaire, pratiques coutumières, littérature orale. Chacun des thèmes abordés fait l'objet d'une synthèse historique qui situe dans le temps et dans l'espace les phénomènes étudiés. Ces synthèses sont d'une grande efficacité. Succinctes et concises, elles sont indispensables pour saisir le sens et la portée des traces " visibles et concrètes » relevées lors des enquêtes sur le terrain. C'est d'ailleurs principalement par le biais de celles-ci que les aspects relevant de la vie sociale et spirituelle des Franco-Ontariens sont véritablement abordés. Le dixième chapitre présente la démarche méthodologique, soit l'élaboration des dossiers ethnohistoriques, la constitution des cahiers de terrain et, finalement, la saisie des données dans une banque informatisée.

Dès le premier chapitre, celui portant sur le patrimoine agricole, le rédacteur de l'ouvrage fait une mise en garde on ne peut plus explicite: 
« Nous avons donc choisi d'orienter presque essentiellement nos enquêtes sur le terrain vers le bâti agricole, l'aspect peut-être le plus évident ou le plus concret ». Ce parti est évidemment déterminant par rapport aux résultats attendus. L'inventaire permet de décrire et de documenter uniquement l'objet sur lequel il porte. Il suffit de parcourir le livre rapidement pour comprendre que, pour l'essentiel, l'enquête de terrain a privilégié le patrimoine immobilier de préférence aux autres facettes du patrimoine franco-ontarien. Ainsi dans le deuxième chapitre consacré au patrimoine religieux, on trouve un bref aperçu du mobilier et des œuvres d'art contenus dans les églises, mais la préoccupation première demeure l'architecture et des éléments d'architecture comme les caveaux, stèles, calvaires, croix, grottes, niches et croix de chemin. Ces éléments sont bien sûr autant d'indices de l'occupation des FrancoOntariens sur le territoire, mais leur analyse reste à faire. Le troisième chapitre traite de l'architecture civile domestique. On y donne « un premier aperçu de l'habitation des Franco-Ontariens demeurant non seulement à la campagne, mais aussi au cœur des villes et des villages ». Une attention particulière est portée aux maisons bourgeoises, aux maisons de « quartiers et d'ouvriers » ainsi qu'aux maisons de compagnies. Les considérations formelles et volumétriques l'emportent sur les aspects liés aux paysages et à l'environnement. L'inventaire ne semble pas fournir beaucoup d'informations sur leur distribution dans l'espace et sur la façon d'occuper le territoire. Le chapitre s'ouvre sur un questionnement: "Existe-t-il une architecture traditionnelle de type villageois ou urbain en Ontario français ? » On laisse entendre que la réponse se trouve dans la compilation systématique des données d'enquête mais que d'ores et déjà des «éléments de réponse commencent à émerger ». La question posée est-elle la bonne ? Ne devraiton pas plutôt s'interroger sur une possible influence des Franco-Ontariens sur l'architecture domestique des territoires qu'ils ont occupés?

La même question se pose pour le patrimoine «commercial et de services » dont traite le chapitre quatrième. Les éléments dont il est question dans ce chapitre comprennent les magasins, les boutiques d'artisan et les hôtels. Outre des considérations portant sur les modes de construction, le discours s'ouvre ici sur des aspects relatifs au rôle qu'ont joué ou jouent encore les Franco-Ontariens dans la collectivité. La présence de ces bâtiments au cœur des villes et des villages témoigne des professions qu'ils ont exercées (commerçants, hôteliers, artisans des métiers du fer, du bois, de la pierre, du cuir et de l'argile) et de l'importance de leur contribution au développement économique local et régional. On aurait pu accoler au chapitre portant sur les commerces et les services celui qui traite du patrimoine industriel, mais on a préféré introduire immédiatement un chapitre portant sur le domaine public ou communautaire. Dans le chapitre cinq, en effet, « On [...] aborde six 
grandes thématiques : les écoles, les hôpitaux, le domaine politique et judiciaire, les transports et communications, le secteur économique et les loisirs et divertissements ». Écoles, couvents, collèges, hôpitaux, hôtels de ville, palais de justice, banques, caisses populaires, gares, bureaux de poste, salles paroissiales, musées sont autant d'éléments du patrimoine collectif qui ont joué un rôle déterminant dans la survie culturelle, sociale, politique et économique des francophones de l'Ontario. Pour le secteur des transports et des communications, on peut s'étonner de ne trouver aucune information sur les ponts, les routes et les voies publiques ainsi que les aéroports. L'inventaire du patrimoine industriel, objet du sixième chapitre, a été subdivisé en quatre principaux secteurs d'activités: l'exploitation des richesses naturelles (industrie minière, industrie forestière), l'agro-alimentaire (brasserie, distillerie, meunerie, minoterie, moulin à farine, beurrerie et fromagerie, manufacture de tabac), le textile et le vêtement (moulin à carder, manufacture de vêtements, manufacture de cuir et de fourrure) et le secteur du transport et des communications (manufacture automobile, construction navale, construction ferroviaire, avionnerie, presse et imprimerie). Surtout " associés au monde rural, au travail en forêt et dans les mines » et ne possédant pas « les clefs du pouvoir économique et politique », la contribution des Franco-Ontariens au développement industriel de la province demeure modeste en dehors du secteur forestier et du secteur agro-alimentaire où « nombre d'entrepreneurs canadiens-français ont pu prendre leur part de marché ». Des scieries, des minoteries, des fromageries ayant appartenu à des familles d'origine francophone témoignent encore aujourd'hui de ces activités en dépit du fait que la plupart des bâtiments qui subsistent ont changé de vocation. Les sources historiques font parfois référence à des industriels franco-ontariens qui se sont distingués dans des secteurs comme l'industrie du vêtement, notamment dans le domaine du cuir et de la fourrure, et dans le secteur manufacturier des voitures : carrioles, traîneaux, charrettes et voitures pour enfants.

Le chapitre sept porte sur l'art populaire. On y présente quelques fabricants de modèles réduits et des sculpteurs qui, à travers leur production, nous font découvrir la vie quotidienne des premiers colons. Les pratiques coutumières sont abordées au chapitre huit, mais les informations qu'on y trouve ne résultent pas des travaux d'inventaire : « nous n'avons guère eu le temps de faire des enquêtes ethnologiques de façon spécifique sur le champ coutumier » nous apprend le rédacteur qui a plutôt choisi d'illustrer certaines d'entre elles en présentant une douzaine de textes tirés d'ouvrages déjà publiés. L'oralité ayant été abondamment documentée par les travaux des folkloristes, dont ceux du père Germain Lemieux (Les vieux m'ont conté) et ceux du professeur Jean-Pierre Pichette de l'Université de Sudbury (Répertoire 
ethnologique de l'Ontario français), l'inventaire ne s'est pas attardé, non plus, à cet aspect du patrimoine franco-ontarien. Le chapitre neuf présente toutefois quelques légendes et quelques chansons provenant de recueils et d'anthologies. La démarche méthodologique sur laquelle repose l'enquête n'est présentée qu'à la toute fin de l'ouvrage, dont elle constitue le dixième et dernier chapitre. Outre les arguments évoqués pour justifier les choix effectués au moment de lancer l'enquête et des informations sur la banque de données qui a servi de fondement à la publication, on y trouve une carte illustrant les trois grandes régions de l'Ontario (l'Est, le Sud et le Nord) où l'on rencontre des francophones. On peut se demander pourquoi ces informations pourtant essentielles à la compréhension de l'étude n'ont pas été présentées plus tôt.

Ce livre expose on ne peut plus clairement la qualité et la diversité du patrimoine architectural de l'Ontario français. L'inventaire aura fait la démonstration de l'importance et de la richesse de son contenu. Mais le produit de l'inventaire et l'ouvrage synthèse qui en découle répondent-ils à la question fondamentale que pose le titre même de la publication? Celle-ci peut se formuler de la façon suivante : en quoi cet inventaire rend-il compte du caractère particulier de l'héritage transmis par les Canadiens français, et en quoi les différents éléments qui composent ce patrimoine témoignent-ils de leur présence en Ontario ? Par définition, le patrimoine est identitaire, il est l'expression même des communautés culturelles, des façons « d'habiter le pays ». En choisissant d'axer l'inventaire sur le repérage des immeubles, les responsables du projet rendaient plus ardue une lecture globale et cohérente de la contribution des Franco-Ontariens au patrimoine de la province. En région urbaine comme en région rurale, les paysages portent la marque des gens qui les habitent et les exploitent. C'est l'activité humaine qui les façonne et les organise en fonction des besoins de la collectivité. L'utilité de l'inventaire est précisément de permettre de lire ces paysages à partir des traces relevées lors des enquêtes de terrain. Ces traces, l'ensemble de ces traces et non seulement le bâti, sont l'une des clés qui donnent accès à une meilleure connaissance des communautés qui ont construit le pays. Dans sa conclusion, le rédacteur de l'ouvrage faisait justement remarquer que «l'objet est [...] porteur de sens, de représentations, d'évocations et de symboliques multiples et composites qui en disent long sur la culture matérielle et spirituelle des communautés humaines ». En privilégiant l'objet immobilier au détriment des autres formes de patrimoine, on a forcément limité la portée de son enquête et par conséquent la lecture qu'on pouvait en faire. En fait, il semble que les informations les plus pertinentes, permettant de saisir l'importance du rôle joué par les Canadiens d'origine francaise en Ontario, proviennent davantage des sources historiques que de l'inventaire proprement dit. Le projet initial 
visait à documenter l'ensemble de la vie matérielle, sociale et spirituelle de l'Ontario français. Ce projet était peut-être trop ambitieux pour les moyens dont disposaient ses concepteurs, mais on ne peut que s'étonner qu'une telle entreprise, conduite sur le terrain par un ethnologue, ait fait aussi peu de place au principal acteur de l'héritage franco-ontarien : l'homme. L'homme dans son milieu, dans ses activités et dans ses gestes, dans ses réalisations et ses paroles, dans l'expression de son savoir et de son savoir-faire. L'homme, en effet, est le grand absent de cet ouvrage.

Bernard Genest

Ministère de la Culture et des communications, Québec 